\title{
Traumatismo torácico y anestesia
}

\section{Thoracic trauma and anesthesia}

\author{
Mauricio Ramos Garrido'
}

\begin{abstract}
The thoracic trauma patient is generally of young, working age, and is often affected by multiple trauma. In its preoperative management, the functional repercussions secondary to thoracic injuries (pulmonary contusion, myocardial contusion, flail chest, etc.) should be evaluated. These become more important when anesthesia must be administered for surgical resolution of neurological, abdominal or limb injuries, and especially, when emergency thoracic surgery must be performed. Only $15 \%$ of patients with chest trauma require surgery. In emergency situations, regional techniques are limited to be a part of the postoperative analgesia or they are indicated in case of extensive nonsurgical parietal lesions. These are patients that have a full stomach in whom, in many cases, a chest drain must be installed prior to or during anesthetic induction. Induction agents that produce less hemodynamic compromise and maintenance with halogenated gases are suggested. Double-lumen endobronchial tubes are the standard method for providing lung isolation. Intraoperative mechanical ventilation should be provided considering the concepts of pulmonary protection taken from intensive medicine. Good postoperative analgesia facilitates respiratory physiotherapy, prevents deterioration of lung function, and allows early ambulation, helping altogether to reduce the incidence of pulmonary and thromboembolic complications.
\end{abstract}

\section{RESUMEN}

El paciente con un traumatismo torácico es generalmente joven, en edad laboral y se presenta habitualmente como un politraumatizado. En su manejo preoperatorio se deben evaluar las repercusiones funcionales secundarias a las lesiones torácicas (contusión pulmonar, contusión miocárdica, tórax volante, etc.). Éstas adquieren mayor importancia cuando debe administrarse anestesia para la resolución quirúrgica de las complicaciones de orden neurológico, abdominal o de extremidades y, especialmente, cuando se debe llevar a cabo una cirugía torácica de urgencia. Sólo 15\% de los pacientes con traumatismo torácico requiere cirugía. En situaciones de urgencia, la anestesia regional está limitada a formar parte de la analgesia post-operatoria o en lesiones parietales extensas. Se trata de pacientes con estómago lleno en los cuales en muchas oportunidades se debe instalar un drenaje torácico previo o durante la inducción anestésica. Se prefiere los agentes de inducción que producen menos compromiso hemodinámico y la mantención de la anestesia con gases halogenados. La intubación monobronquial debe ser prácticamente rutinaria. La ventilación mecánica

Hospital de Urgencia Asistencia Pública, Instituto Nacional del Tórax.

Fecha de recepción: 21 de noviembre de 2020

Fecha de aceptación: 29 de noviembre de 2020

ORCID

0000-0002-7801-2744

Correspondencia:

Dr. Mauricio Ramos

mauroramosg@gmail.com 
intraoperatoria debe ser manejada con los conceptos de protección pulmonar provenientes de la medicina intensiva. Una buena analgesia posoperatoria facilita la kinesiterapia respiratoria, evita el deterioro de la función pulmonar y permite una deambulación precoz, todo lo cual contribuye a disminuir la incidencia de complicaciones pulmonares y tromboembólicas.

E n la población general de Europa y Estados Unidos, los accidentes con trauma severo constituyen la cuarta causa de muerte y la primera en menores de 34 años. La principal fuente de origen de lesiones torácicas son los accidentes de tránsito, habitualmente asociados a la ingesta de alcohol y a la conducción nocturna o desaprensiva[1]. La conducción a alta velocidad tiene relación con la severidad y morbimortalidad del traumatismo[2]. Las lesiones torácicas están presentes casi en la mitad de los choferes que conducen sin cinturón de seguridad al momento del accidente.

Se trata en general de pacientes jóvenes en edad laboral, predominantemente de sexo masculino. Las lesiones traumáticas torácicas están frecuentemente asociadas a lesiones en otra localización (60\%-70\%), las que frecuentemente marcan el pronóstico y es por ello que habitualmente el cuadro clínico forma parte de un politraumatismo, dentro del cual se debe tratar el traumatismo torácico[1].

Un tercio de los pacientes hospitalizados por traumatismo presenta compromiso del tórax, que potencialmente puede dañar un número importante de órganos vitales, lo que explica su gravedad. En politraumatizados, el traumatismo torácico cerrado es responsable de un cuarto de las muertes[3] y contribuye en otro cuarto de ellas[4]. Muchas de las causas son potencialmente tratables como el taponamiento cardíaco, el neumotórax a tensión o la obstrucción de la vía aérea. Un cuarto de los pacientes es de gravedad en el momento del ingreso y otro cuarto se agrava en el transcurso de la hospitalización.

\section{Mecanismos del trauma torácico}

Para la producción de un traumatismo se requiere de una cierta energía (cinética) descargada sobre la pared torácica, produciendo variaciones de presión sobre ésta y las estructuras subyacentes. Los distintos mecanismos involucrados junto a la gran variedad de órganos contenidos en la caja torácica explican la diversidad de las lesiones producidas, las cuales no son todas de resolución quirúrgica.

Los mecanismos mencionados son:

- Traumatismo directo: lesiona en forma directa el lugar de impacto y actúa además por contragolpe. - Desaceleración: produce desplazamiento de órga- nos (torácicos y abdominales). Aquellos de mayor masa, son capaces de actuar sobre aquellos de menor tamaño y/o menos densos en su estructura $y$, por otra parte, pueden desprenderse de su anclaje anatómico normal.

- Onda expansiva: la onda de presión propagada provoca un daño primario directo al momento de hacer contacto con estructuras que tienen interface aire/tejido, como ocurre en los sistemas auditivo, pulmonar y gastrointestinal[5].

- Heridas: la severidad de las lesiones producidas por arma de fuego y por arma blanca dependerá del tipo de dispositivo y de su trayectoria. Las heridas penetrantes a alta velocidad son responsables de una destrucción torácica mayor.

\section{Lesiones parietales}

Alrededor de tres cuartas partes de los pacientes con traumatismo torácico presentan fracturas de los elementos óseos de la caja torácica[1]. Por otra parte, las fracturas costales están presentes en $10 \%$ de los pacientes politraumatizados[6].

\section{Fracturas costales}

Las costillas más frecuentemente involucradas son desde la $5^{a}$ a la $9^{a}$. La lesión de las dos primeras costillas implica una violencia muy importante, por ello es que según algunos autores esto es sugerente de la existencia de lesiones nerviosas y vasculares, lo que no ha sido comprobado por los estudios. Las fracturas de las costillas más bajas $\left(8^{\mathrm{a}}\right.$ a $\left.12^{\mathrm{a}}\right)$ se asocian a lesiones abdominales altas, constituyéndose un traumatismo toracoabdominal.

La mortalidad y las complicaciones pulmonares se asocian al número de costillas lesionadas[7], la presencia de comorbilidades y la edad[8]. En los pacientes de mayor edad se requiere menos energía para la producción de lesiones parietales[9]. Esto se ve reflejado en series donde los pacientes mayores de 65 años con fracturas costales presentan mayor morbilidad, su estadía intrahospitalaria es más prolongada (15 vs 10 días) y tienen el doble de la mortalidad que los pacientes más jóvenes con lesiones de magnitud similar[8]. 


\section{Tórax volante}

El tórax volante se produce por la fractura de tres o más costillas que tengan dos o más rasgos de lesión, conformando un área de la pared torácica independiente del resto, que se mueve en forma paradojal con los movimientos respiratorios. Su localización puede ser lateral, anterior, esterno-costal o combinaciones de estos tipos y presentarse a distinta altura de la caja torácica. Dado que requiere una gran energía, existe una alta probabilidad de asociación a otras lesiones torácicas, abdominales o neurológicas. Aún cuando se trata de un cuadro muy grave su mortalidad es menor que lo esperado, situándose en la actualidad en alrededor del 5,6\%[10].

La unión del tercio posterior con el tercio medio de la costilla es un límite de estabilidad que puede ayudar a individualizar los casos con mayor riesgo de compromiso funcional. De este modo, existe un importante riesgo de inestabilidad si las fracturas se sitúan por delante del tercio posterior (tórax volante anterior) o bajo la $6^{a}$ costilla. Por el contrario, si las fracturas se encuentran sobre la sexta costilla y su rasgo posterior está por detrás del tercio medio (tórax volante posterior) se estima que son más estables; del mismo modo, si la distancia entre ambos rasgos es corta hay mayor estabilidad y, por el contrario, si la distancia es larga la estabilidad disminuye.

Hay varios factores que pueden contribuir a una insuficiencia respiratoria aguda en este cuadro: hipoventilación, acumulación de secreciones debido a la falta de tos como mecanismo antiálgico o, en un tercio de los casos, una contusión pulmonar subyacente.

Durante un tiempo se usó la ventilación mecánica como parte de la estabilización del tórax, sin embargo, demostró ser innecesaria en la mayoría de los casos, aumentando además el tiempo de hospitalización debido a sus complicaciones. La ventilación mecánica debe instituirse en forma discriminada y no de rutina. Hay pacientes que nunca requieren ventilación mecánica, mientras otro grupo de pacientes decididamente la necesitan por su lesión torácica o por la presencia concomitante de traumatismo encéfalocraneano y hay un grupo de pacientes en una situación intermedia en los cuales la decisión debe ser tomada según la presencia de lesiones extratorácicas y sus consecuencias funcionales. Existen publicaciones que han propuesto el manejo con ventilación mecánica no invasiva[11].

En algún momento se planteó que la inestabilidad parietal era el mecanismo principal de la insuficiencia respiratoria aguda por lo que se consideró preponderante la osteosíntesis costal y/o esternal en su manejo. Sin embargo, este tratamiento quirúrgico se fue limitando a casos extremos o a aquellos en que existía alguna otra indicación quirúrgica. Con la aparición de nuevos elementos de osteosíntesis que han simplificado la técnica, ha resurgido el entusiasmo por la resolución quirúrgica precoz y, aún cuando los resultados han sido criticados en alguna oportunidad, ${ }^{12}$ continúan apareciendo estudios en que se argumentan beneficios de esta cirugía[13],[14].

El alivio del dolor y la limpieza (toilette) bronquial son primordiales en el manejo de las lesiones parietales. Los opioides sistémicos son de primera línea en aquellos pacientes que requieren intubación y ventilación mecánica, sin embargo, en pacientes no intubados tienen efectos adversos como depresión respiratoria, supresión de la tos y sedación, tres condiciones que pueden llevar a la formación de atelectasias, retención de secreciones, disminución de la capacidad residual funcional y empeoramiento de la hipoxia[6],[9],[15],[16].

Las técnicas de analgesia regional tienen la ventaja de no producir depresión central mostrando en contrapartida un efecto analgésico claramente superior, lo que se traduce en un mejor resultado[17],[18],[19].

\section{Fractura esternal}

La fractura esternal es un marcador de la violencia del trauma sufrido. Su frecuencia de presentación alcanza al 1,5\% a $4 \%$ de los traumatismos toráci$\cos [20],[21]$. El $2 \%$ de los individuos involucrados en accidentes de tránsito presenta una fractura de esternón y como factores de riesgo relativo se mencionan la edad sobre los 50 años y el sexo femenino en mayores de 50 años (Tabla 1). El uso del cinturón de seguridad puede contribuir al aumento en la presentación de esta fractura.

Normalmente, es de rasgo transversal, comprometiendo en el $93 \%$ de los casos el cuerpo del esternón y habitualmente $(70 \%)$ el desplazamiento es mínimo o nulo. La fractura del manubrio implica un mayor grado de violencia. Se ha insinuado una asociación entre la altura de la fractura esternal y la presencia de lesiones cervicales asociadas.

Es una lesión que tradicionalmente se ha asumido como seria y de compromiso vital. Sin embargo, un estudio retrospectivo sobre 272 pacientes mostró que la trascendencia de esta lesión es menor a la que se pensaba originalmente. ${ }^{20}$ No todos los pacientes requirieron hospitalización y muchos de los que fueron admitidos tuvieron una estadía corta (menos de 3 días), prolongándose sólo en los pacientes añosos o en quienes la etiología implicaba caída o golpe di- 
recto. Solamente el 1,5\% de los pacientes presentó arritmias que requirieron tratamiento. La mortalidad observada fue sólo de $0,74 \%$ (sin fractura esternal: $4,9 \%$ ) muy por debajo de la descrita en trabajos previos (Tabla 2)[22],[23],[24] Estos resultados Ilaman la atención ya que esta fractura tradicionalmente hace sospechar la existencia de lesiones intratorácicas, que se presentan en algo más de la mitad (55\%) de los pacientes.

Una de las posibles complicaciones de esta lesión es el hematoma mediastínico, que obliga a descartar otras posibles causas. ${ }^{25}$ Este hematoma puede comprimir estructuras mediastínicas y/o el pulmón, lo que puede producir insuficiencia respiratoria.

\section{Fractura de escápula}

También se requiere un impacto de alta energía para fracturar la escápula. En un estudio retrospectivo de 10 años[26], que incorporó 35.541 traumas cerrados, hubo una incidencia de 1,1\% $(n=392)$. El $99 \%$ de estos 392 pacientes con fractura de escápula presentaron alguna lesión asociada, pero sólo el 1\% de ellos presentó una lesión de aorta.

\section{Lesiones pulmonares}

Más de las tres cuartas partes de los casos son de ubicación unilateral y, a pesar de su alta frecuencia (33\%), su diagnóstico no siempre es fácil. La severidad de las lesiones parietales no es un índice fiel de la existencia de lesiones pulmonares y, por otra parte, la radiografía de tórax no es sensible ni específica.

\section{Onda expansiva}

El pulmón recibe en su interior la energía sobre la interfase aire líquido. Toda vez que se sospeche una lesión de este tipo debe buscarse concomitantemente una ruptura timpánica[1]. Existe la posibilidad de embolia gaseosa arterial por lo cual, cuando sea posible, se debe mantener al paciente en ventilación espontánea (presión negativa) o bien, en ventilación monopulmonar contralateral, con $\mathrm{FiO}_{2}$ de $100 \%$ para disminuir el tamaño de los émbolos.

\section{Hematoma intrapulmonar}

Corresponde a una ruptura pulmonar central, que en general regresa en forma espontánea en semanas.

\section{Pseudoquiste postraumático o neumatocele}

Se trata de una lesión pulmonar más rara. Se produce después de un traumatismo menor en pacientes jóvenes o niños. Aunque la radiografía puede mostrarlo inmediatamente en algunos casos, lo habitual es que se haga evidente a la primera o segunda semana. Regresa espontáneamente en algunas semanas.

\section{Laceración o ruptura pulmonar}

Una lesión de vasos pulmonares normalmente se cohibe en forma espontánea en virtud de la baja presión en el circuito vascular pulmonar. Un estudio de

Tabla 2. Tasas de mortalidad de pacientes con fractura esternal según diferentes publicaciones[20]

\begin{tabular}{lcc}
\hline Autor & Año & Tasa \\
Gibson & 1962 & $19 / 80$ \\
Breederveld & 1988 & $3 / 71$ \\
Maxwell & 1988 & $3 / 51$ \\
Wojcik & 1988 & $0 / 66$ \\
Otremski & 1990 & $16 / 78$ \\
Brookes & 1993 & $2 / 272$ \\
\hline
\end{tabular}

\begin{tabular}{lcc} 
& Tabla 1. Riesgo relativo de fractura esternal en accidentes de tránsito[20] \\
\hline & Incidencia (\%) & Riesgo relativo \\
Todos los pacientes & $14 / 703(2,0)$ & 1,00 \\
Todas las mujeres & $9 / 325(2,8)$ & 1,40 \\
Todos los hombres & $5 / 360(1,4)$ & 0,70 \\
$>50$ años & $11 / 118(9,32)$ & $4,68^{*}$ \\
$<50$ años & $3 / 569(0,5)$ & 0,25 \\
Mujeres $>50$ años & $8 / 78(10,26)$ & $5,15^{*}$ \\
Mujeres $<50$ años & $1 / 247(0,4)$ & 0,20 \\
\hline
\end{tabular}

\footnotetext{
* Factor significativo
} 
2001 mostró que la reparación o resección de parénquima pulmonar fue necesaria en menos del $2 \%$ de los casos de trauma cerrado y en menos del $6 \%$ en casos de trauma penetrante[27].

\section{Contusión pulmonar}

En su producción influyen la violencia y la velocidad del traumatismo. Es una lesión evolutiva, con aparición en horas y regresión en días. Se presenta entre el $9 \%$ y $25 \%$ de los traumatismos torácicos cerrados[28], pero esta proporción aumenta (65\%) en los casos que requieren cirugía de urgencia[29].

En los pacientes menores de 40 años la posibilidad de contusión pulmonar es mayor que la de fracturas costales múltiples, a diferencia de los mayores de 60 años en quienes ocurre lo contrario[30]. Esto se debería a que la constitución ósea de los pacientes de mayor edad hace más fácil la fractura costal ante una misma energía, disipándose esta energía e impidiendo que el parénquima pulmonar la reciba.

La contusión pulmonar se asocia a distress, neumonía y muerte, eventos que se relacionan con la magnitud de parénquima pulmonar afectado[31]. La base de las repercusiones funcionales es el aumento de la permeabilidad capilar, sobre la cual actúan factores iniciales como la lesión tisular directa y las alteraciones del surfactante, factores secundarios como la sepsis y la respuesta inflamatoria, y fenómenos de isquemia-reperfusión. Elementos agravantes son la toxicidad por oxígeno y el barotrauma.

El cambio que ocurre en la permeabilidad es generalizado, aunque la lesión inicial haya sido unilateral. Se produce extravasación de líquido rico en proteínas al intersticio, pequeños bronquíolos y espacio alveolar, con lo que se altera aún más el surfactante. Las consecuencias funcionales son alteración de la relación $\mathrm{V} / \mathrm{Q}$, aumento del espacio muerto y disminución de la distensibilidad pulmonar.

La severidad de la contusión es difícil de evaluar en un inicio ya que la hipoxemia inicial no es un buen índice, así como tampoco la radiografía de tórax, que evalúa la zona de alveolitis hemorrágica, pero no el área de edema perilesional. Si bien el deterioro gasométrico se relaciona con la extensión de la contusión, también colaboran las alteraciones de la relación V/Q en el tejido pulmonar adyacente a la contusión misma, ya que en el área contundida se produce una disminución de la perfusión en respuesta a la disminución en la ventilación. Si bien la tomografía es el gold standard como método diagnóstico, la ecografía puede contribuir más rápidamente a la evaluación y diagnóstico[32],[33].
En su tratamiento es importante la analgesia y deben evitarse injurias secundarias como aspiración, embolia, sepsis, daño inducido por ventilación mecánica ${ }^{34}$ y aporte excesivo de volumen (politransfusión, balance hídrico positivo). Esta patología tampoco es sinónimo de ventilación mecánica y la decisión debe ser guiada por la función pulmonar y la aplicación de criterios clásicos.

\section{Hemotórax/neumotórax}

El diagnóstico de neumotórax se hace en base a una radiografía, tomografía o ecografía. Esta última es un método diagnóstico cada vez más usado por su facilidad de realización y buena sensibilidad en la detección de neumotórax y hemotórax[35],[36].

En todo paciente traumatizado que presente un neumotórax debe instalarse una pleurostomía en base a tres consideraciones: la eventual necesidad de ventilación a presión positiva que puede derivar en un neumotórax a tensión, el riesgo de compresión del pulmón contundido subyacente favoreciendo la producción de atelectasias y, la asociación a un hemotórax de poca cuantía.

El hemotórax también debe ser drenado como medio de evaluación o descarte de la persistencia del sangrado inicial. El hemotórax masivo es producido, en frecuencia decreciente, por: arteria intercostal, arteria mamaria interna, hilio pulmonar, vaso mediastínico, ruptura cardíaca o de istmo aórtico.

Lo habitual es la evacuación de un hemoneumotórax moderado bien tolerado, por medio de un drenaje pleural. El abordaje quirúrgico está indicado cuando se presenta una evacuación inicial mayor a un litro o un débito de $200 \mathrm{ml} \cdot \mathrm{h}^{-1}$ por $4 \mathrm{~h}[37]$.

En estos casos el abordaje por videotoracoscopía implica menos tiempo de estadía hospitalaria y menores tasas de infección[38]. Algunos centros manejan como norma realizar una videotoracoscopía a todos los pacientes con hemotórax independiente de su cuantía, para realizar una evacuación completa evitando la posible organización de la sangre residual con sus alteraciones mecánicas y eventual sobreinfección, además de solucionar inmediatamente las lesiones que eventualmente se identifiquen[39].

\section{Ruptura diafragmática}

La ruptura diafragmática es consecuencia de un traumatismo toracoabdominal. Su localización es posterolateral (porción tendinosa), con trayecto radial y de longitud variable. Las heridas penetrantes producen rupturas más localizadas, en tanto que la compre- 
sión torácica produce avulsión de las uniones costales.

La ventilación espontánea a presión negativa puede provocar el desplazamiento de órganos abdominales hacia la cavidad torácica (estómago, colon izquierdo, bazo, mesenterio, intestino delgado, lóbulo hepático izquierdo), con la posibilidad de incarceración y estrangulación, que es más probable mientras menor sea la solución de continuidad del diafragma. Como consecuencias se puede producir respiración paradojal, compresión del pulmón por los órganos herniados y compresión de mediastino, dificultando el retorno venoso.

\section{Lesiones miocárdicas}

La contusión miocárdica es una entidad que puede presentarse en un espectro desde petequias hasta la ruptura miocárdica, lo que explica su amplia variabilidad de incidencia (0\% a $76 \%$ ) en pacientes con traumatismo torácico de acuerdo a la metodología utilizada para su diagnóstico[40]. La contusión es principalmente de ventrículo derecho y, con mucho menor frecuencia, ocurren lesiones de válvulas, septum interventricular, tejido de conducción o arterias coronarias.

Su diagnóstico de certeza es difícil. Clínicamente se logra este diagnóstico en 5\% a 15\% de los pacientes que lo presentan[3]. Si la situación clínica es evidente no se requiere de métodos sofisticados suplementarios. Por otra parte, establecer claramente el diagnóstico de contusión miocárdica en pacientes hemodinámicamente estables no es de gran importancia toda vez que el riesgo de morbilidad significativa en ellos es mínimo[40].

Algunas publicaciones señalan que si el ECG de ingreso es normal hay muy baja probabilidad de que exista alguna injuria miocárdica[41]. El ECG puede mostrar alteraciones del ritmo (extrasístoles, bloqueos de rama o auriculoventriculares) que normalmente no son de riesgo vital[42], así como alteraciones en la repolarización que se manifiestan como cambios en el segmento ST y la onda T[43].

La ecocardiografía detecta alteraciones en la motilidad segmentaria o la presencia de derrame pericárdico. También puede evidenciar daño de estructuras, pero esto es mucho menos frecuente. La técnica transesofágica es más sensible que la transtorácica en la detección de contusión miocárdica, lesiones valvulares o de aorta torácica[44],[45].

Otros métodos diagnósticos a los cuales se puede recurrir son diversos. La radiografía de tórax y la fracción miocárdica de la creatinfosfoquinasa son poco sensibles, así como también la cintigrafía con Tc 99.
La cintigrafía con Talio 201 tiene limitaciones por su incapacidad de estimar la lesión de ventrículo derecho, justamente el más expuesto. La determinación plasmática de componentes de la célula miocárdica (troponina, miosina) muy útiles en el diagnóstico de infarto al miocardio ha demostrado una pobre sensibilidad en este contexto[40],[46].

Si bien está descrito que esta patología puede conducir a compromiso hemodinámico, falla del ventrículo derecho con hipertensión pulmonar o incluso paro cardiaco[47], es raro que esto ocurra[48].

La ruptura miocárdica generalmente se debe a una herida penetrante por arma blanca o de fuego. Las lesiones producidas por armas de fuego tienden a ser más difusas y a inducir lesiones de cavitación, por lo que en general son más graves que las producidas por arma blanca. La indicación quirúrgica de las heridas penetrantes no tiene discusión en aquellos casos que tienen compromiso hemodinámico. Por el contrario, las heridas penetrantes asintomáticas, con radiografía y/o ecografía torácica normales, deben ser manejadas con conducta expectante.

El trayecto determina la(s) cavidad(es) o estructuras lesionadas. El $40 \%$ de los casos presenta compromiso de ventrículo derecho, $24 \%$ de aurícula derecha, $40 \%$ de ventriculo izquierdo, $5 \%$ lesión coronaria (normalmente la arteria descendente anterior), pudiendo haber también lesiones del septum interauricular o interventricular o válvulas[49]. Esta misma publicación reporta una mortalidad por arma blanca de $52 \%$ y por arma de fuego de $42 \%$. En contraposición, un reporte de 2006, demostró una mortalidad de $81 \%$ en caso de lesión por arma de fuego, y una mortalidad de sólo 15\% en caso de lesión por arma blanca; destaca, además que el taponamiento cardiaco (53\% de los casos) se asocia a una mortalidad de sólo 8\%[50].

\section{Lesiones vasculares}

Todas las estructuras vasculares dentro del tórax pueden ser alcanzadas por el trayecto de un arma blanca o de fuego, provocando lesiones arteriales o venosas que tienen un carácter de mucha gravedad. En casos de traumatismo torácico cerrado se activan otros mecanismos que provocan lesiones en el árbol arterial, principalmente la aorta o los vasos supraaórticos.

La ruptura de los vasos supraaórticos ocurre por hiperextensión, rotación cervical y también por compresión anteroposterior (primera costilla). La frecuencia con que se presentan estas lesiones en or- 
den decreciente es: tronco braquiocefálico, subclavia izquierda y carótida primitiva izquierda. Dada la violencia necesaria para este tipo de lesión es frecuente la asociación de ellas, especialmente cuando existe compromiso de la carótida izquierda.

En cuanto al mecanismo de la lesión de aorta torácica se han postulado varias teorías no excluyentes:

- Desaceleración con tracción del istmo en relación al ligamento ductal (Figura 1).

- Compresión del tórax con aproximación de estructuras óseas, lo que también produce tracción del istmo.

- Impacto abdominal o torácico bajo que produce desplazamiento craneal de órganos del mediastino con aumento de tensión sobre la aorta descendente proximal.

- Impacto directo que produce un aumento agudo en la presión intraaórtica.

Su mortalidad inicial es muy alta, alcanzando alrededor de $85 \%$ en el lugar del accidente[51]. Los pacientes que sobreviven, lo hacen gracias a la formación de un hematoma perilesional que limita la hemorragia, o bien, por la formación de un falso aneurisma (adventicia indemne) cuya ruptura secundaria se puede producir desde horas a días después o evolucionar hacia su organización. De todas formas, los pacientes que alcanzan a llegar al hospital presentan una mortalidad de $50 \%$ principalmente debido a las lesiones asociadas[52].

El sitio clásico de ruptura es el istmo, lo que puede deberse a un factor congénito o a un factor mecáni$\mathrm{co}$, ya que la aorta se vuelve relativamente fija a partir del ligamento arterioso que es donde se une a la arteria pulmonar izquierda, quedando móvil la parte más proximal de la aorta torácica que es susceptible de desplazarse y angularse. La mayoría de las rupturas aórticas presentan un rasgo lineal, transverso, con compromiso de las tres capas de la pared vascular y de una porción mayor de la circunferencia aórtica.

La ruptura traumática del istmo aórtico tiene una incidencia de $12 \%$ a $15 \%$ en las autopsias por accidente de tránsito y corresponde a $70 \%$ a $90 \%$ de las lesiones graves de vasos del mediastino (Figura 1). Un estudio de Feczko y cols, realizado en 142 autopsias por accidente de tránsito con lesión aórtica mostró que el $70 \%$ eran hombres (edad media: 37,5 años) y $30 \%$ mujeres (edad media: 46 años), con una mortalidad de $62 \%$ en los primeros 30 minutos; el sitio más frecuente de ruptura fue el istmo aórtico, inmediatamente distal al nacimiento de la subclavia izquierda en $54 \%$ de los casos, existiendo un $18 \%$ de casos con más de un punto de lesión[53].

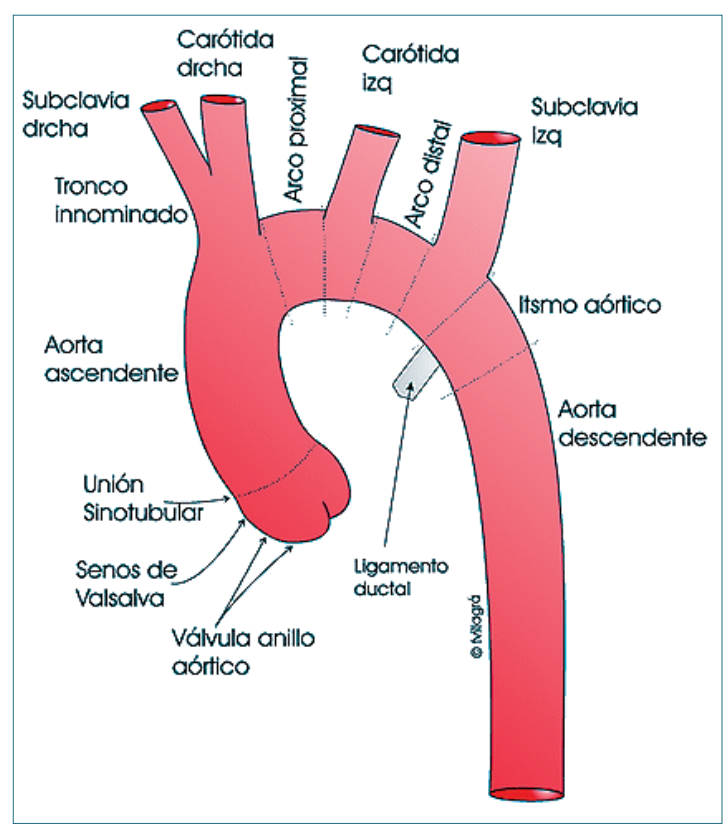

Figura 1. Ubicación del istmo aórtico en relación con el arco aórtico.

En pacientes operados se identificó la ruptura en la aorta descendente en el 93\%, en la aorta ascendente en el $3 \%$, en el arco aórtico en el $4 \%$ y yuxtadiafragmática en el 0,3\% de los casos[54].

Los signos externos de traumatismo no siempre son concordantes con el daño interno[53],[55] ya que $36 \%$ de los pacientes presentan signos menores de traumatismo externo. Aunque se ha mencionado la asociación con las fracturas de primera y segunda costillas no existe correlación con las lesiones esqueléticas: las fracturas costales tienen un despreciable valor predictivo positivo de $15 \%$ y una especificidad de sólo 57\%[56].

El estudio multicéntrico de Fabian y cols., que incluye 274 pacientes[54], muestra que la lesión aórtica se asocia a un gran número de otras lesiones (Tabla 3). La radiografía de tórax sigue siendo el examen inicial más usado. En la Tabla 4 se describen los hallazgos de la radiografía de ingreso, que puede ser normal en el $28 \%$ de los casos, haciéndose positiva algunas horas después[57]. Es destacable que sólo el $12 \%$ a $18 \%$ de los hematomas mediastínicos se debe a ruptura aórtica[58].

El examen que clásicamente se ha considerado de certeza es la angiografía, tanto para definir el diagnóstico como el lugar de la ruptura. En el ambiente bélico las imágenes de radiografía, tomografía o angiografía son el medio diagnóstico más utilizado[59],[60],[61]. 
Dado que el paciente politraumatizado es frecuentemente derivado al servicio de radiología para la realización de una tomografía computarizada para la evaluación de un posible TEC y/o trauma abdominal, ésta puede ser usada también para la evaluación de la aorta torácica. En un inicio fue catalogada como un mal método por presentar, en relación con la aortografía, una sensibilidad de sólo 55\% a $83 \%$ y una especificidad de $23 \%$ a $65 \%[62],[63]$, con $17 \%$ de falsos negativos y $39 \%$ de falsos positivos[64]. Sin embargo, el desarrollo tecnológico de la tomografía computarizada ha permitido mejorar su rendimiento[16] a una positividad de $74 \%$ (23\% equívoca, 3\% negativa) y un valor predictivo negativo de $100 \%$ [65].

La tecnología helicoidal permite realizar una tomografía en forma muy rápida y reconstruir imágenes finas, lo que permite evaluar la pared de la aorta torácica. Un estudio demostró una sensibilidad de 100\% versus $92 \%$ de la aortografía: los casos de falsos negativos de la aortografía fueron lesiones mínimas de la íntima sin formación de aneurisma, que muy probablemente no tienen significado clínico[66]. La especificidad de la TAC fue de $83 \%$ debido a la detección de falsos positivos correspondientes a divertículos, en comparación con 99\% de la aortografía. Esto otorgó un valor predictivo positivo de $50 \%$ para el scanner helicoidal y un valor predictivo negativo de 100\% versus $97 \%$ para ambos valores predictivos para la aortografía. Estos resultados lo convierten en un examen de mucha utilidad en el estudio de pacientes con diagnóstico probable de lesión vascular secundaria a un traumatismo de tórax.

La ecocardiografía transesofágica es un método diagnóstico muy valioso[67]. Sin embargo, no está disponible en todos los centros por tratarse de un equipo sofisticado y porque se necesita un operador entrenado. La limitación principal de esta técnica es que no se visualiza adecuadamente el arco aórtico ni sus ramas principales[21].

La ruptura aórtica es normalmente una emergencia quirúrgica por ser una causa de muerte inminente. El estudio de Fabian[54] incluyó 274 pacientes con lesión aórtica por trauma cerrado. Esta serie tuvo una mortalidad global de $31 \%$, pero aquellos pacientes que fueron operados en condiciones estables tuvieron solamente $14 \%$ de mortalidad. Por otra parte, los 21 pacientes que no fueron operados tuvieron $52 \%$ de mortalidad. Mientras que aquellos que fueron intervenidos en condiciones hemodinámicas extremas (22) tuvieron $100 \%$ de mortalidad, al igual que el grupo de pacientes (24) en quiénes se completó la ruptura de la aorta (24). La Tabla 5 resume la mortalidad y la Tabla 6 muestra las complicaciones de esta casuística.

La presencia de otras complicaciones graves que afecten al paciente (Tabla 6) puede postergar la in-

Tabla 4. Hallazgos en radiografía de tórax de ingre-
so[54]

\begin{tabular}{lclc}
\hline \multicolumn{4}{c}{ Tabla 3. Lesiones asociadas (\%) a lesión aórtica traumática de la aorta[54] } \\
\hline Lesión & $\%$ & Lesión & $\%$ \\
TEC & 51 & Médula espinal & 4 \\
Fracturas costales & 46 & Pelvis & 31 \\
Tórax volante & 12 & Fémur & 24 \\
Contusión pulmonar & 38 & Tibia & 22 \\
Contusión miocárdica & 4 & Extremidad superior & 20 \\
Ruptura diafragmática & 7 & Maxilofacial & 13 \\
Bazo & 14 & Columna cervical & 4 \\
Hígado & 22 & Columna torácica & 4 \\
Intestino delgado & 7 & Columna lumbar & 4 \\
Otras lesiones abdominales & 14 & & \\
\hline
\end{tabular}


dicación de cirugía inmediata. Ejemplos de esto son la definición del estado neurológico, si existe un TEC asociado, o un hematoma retroperitoneal que puede emporar si el paciente recibe anticoagulación. Sin embargo, retardar la cirugía sobre la aorta no aumenta el tiempo de estadía intrahospitalaria, la morbilidad ni la mortalidad como lo mostró un estudio retrospectivo realizado en 48 pacientes[68], aún cuando este estudio fue realizado para demostrar que no se ganaba nada con retardar la cirugía. Desde el punto de vista económico hubo un aumento de los costos de hospitalización a prácticamente el doble.

La paraplejia es una de las complicaciones más temidas de la lesión aórtica, mencionándose como posibles causas la lesión directa de la médula, lesión de las arterias espinales o hipoperfusión distal al sitio de ruptura[69]. Este daño neurológico se puede detectar en $2,6 \%$ de los pacientes al momento de su ingreso al centro asistencial. Sin embargo, es más frecuente observarla como complicación de la cirugía en la cual se presenta con una frecuencia cercana al $9 \%$.

Entre los factores quirúrgicos importantes están la altura de la lesión y un tiempo seguro de pinzamiento aórtico inferior a 30 min[54],[68] (Tabla 7). Este tiempo puede prolongarse 15 minutos si se mantiene la perfusión distal con bypass aurículo-femoral con bomba centrífuga o circulación extracorpórea fémoro-femoral[54]

Aún cuando no se cuenta con evidencia a largo plazo, la reparación endovascular parece ser un tratamiento efectivo y razonable para la lesión traumática de la aorta torácica[70]. Algunos estudios en que la reparación endovascular se hace en forma diferida muestran una menor mortalidad, incidencia de paraplejia y accidente vascular encefálico[71],[72],[73], mientras que otros no muestran menor mortalidad pero sí menor daño neurológico[74]. Una revisión de 2015 revela que la reparación endovascular diferida tiene beneficios comparativos, incluyendo la disminución de la mortalidad[75], pero un metaanálisis del mismo año no logra demostrar evidencia que dirima las opciones de tratamiento para esta grave lesión[76].

\section{Otras lesiones}

La ruptura traqueo-bronquial es una complicación rara. El lugar de ruptura normalmente es cercano a la carina (fin de la tráquea o inicio de los bronquios fuente). Se presenta con neumotórax, hemoptisis, atelectasia, enfisema mediastínico y subcutáneo. Las lesiones sobre el árbol bronquial deben ser diagnosticadas lo más precozmente posible, ya que después de las 24 h de evolución, presentan mayor riesgo de mortalidad[77].

Se ha propuesto la utilización de ventilación a alta frecuencia para el manejo de la fístula broncopleural o disrupción tráqueo-bronquial, lo que ha sido rebatido por algunos autores. También se ha propuesto la

\begin{tabular}{lc}
$\begin{array}{l}\text { Tabla 6. Complicaciones de un estudio realizado en } \\
\text { 274 pacientes con lesión aórtica[54] }\end{array}$ \\
\hline Complicaciones & $\mathbf{n}$ \\
Neumonía & 68 \\
Insuficiencia renal & 18 \\
Empiema & 4 \\
Absceso abdominal & 10 \\
Paraplejia & 18 \\
Lesión de nervio recurrente & 9 \\
Aneurisma de la sutura & 1 \\
\hline
\end{tabular}

\begin{tabular}{|c|c|c|c|c|c|}
\hline & Estables & No operados & In extremis & Ruptura & Todos \\
\hline$n$ & 207 & 21 & 22 & 24 & 274 \\
\hline Ruptura & 8 & 0 & 22 & 24 & 54 \\
\hline TEC & 6 & 5 & 0 & 0 & 11 \\
\hline FMO & 8 & 5 & 0 & 0 & 13 \\
\hline SDRA & 3 & 1 & 0 & 0 & 4 \\
\hline TEP & 1 & 0 & 0 & 0 & 1 \\
\hline No conocido & 3 & 0 & 0 & 0 & 3 \\
\hline Fallecidos $N^{\circ}(\%)$ & $29(14)$ & $11(52)$ & $22(100)$ & $24(100)$ & $86(31)$ \\
\hline
\end{tabular}

TEC: traumatismo encefalocraneano; FMO: falla multiorgánica; SDRA: síndrome de distress respiratorio del adulto; TEP: tromboembolismo pulmonar. 
Tabla 7. Incidencia de paraplejia según tiempo de pinzamiento aórtico[54]

\begin{tabular}{lccc}
\hline Grupo & Paraplejia & Sin paraplegia & P \\
$<$ de 30 minutos & 1 & 60 & \\
$>$ de 30 minutos & 15 & 109 & 0,02 \\
\hline
\end{tabular}

utilización de ventilación monopulmonar para estos casos, con el uso de ventilación diferencial (ventilación alta frecuencia - ventilación a presión positiva clásica). Su tratamiento incluye algunas técnicas realizadas por broncoscopía y, por supuesto, la cirugía[78].

Las rupturas esofágicas son excepcionales en el caso de un traumatismo cerrado, siendo la causa más frecuente las heridas penetrantes, en especial por arma de fuego.

\section{Manejo inicial del trauma torácico}

Se han publicado diversos puntajes para la toma de decisiones en pacientes politraumatizados que reciben atención prehospitalaria[79],[80]. Uno de los aspectos a definir es la necesidad de intubación prehospitalaria en el contexto del shock hemorrágico, lo cual está refutado por algunos estudios[81], salvo que se acompañe de un deterioro neurológico objetivado a través de un puntaje en la escala de Glasgow de 8 o menos[82].

Las primeras medidas se destinan a evaluar el riesgo vital a que está sometido el paciente, determinando si su causa es de tratamiento quirúrgico o no y a estabilizar las funciones ventilatoria y circulatoria. En esta fase el anestesista puede ser requerido antes de la llegada del paciente a pabellón.

Aparte de las diferentes lesiones descritas anteriormente, se debe considerar también la posibilidad de obstrucción de la vía aérea superior por cuerpo extraño, compromiso por traumatismo maxilofacial o encéfalocraneano o dilatación aguda del estómago. También se deben considerar condiciones que establecen un shunt pulmonar alto como la patología intrapulmonar, embolia (trombótica, grasa o aérea) aspiración de contenido gástrico o distress respiratorio.

El trauma óseo puede originar embolia grasa de mayor o menor cuantía a partir de diversos puntos de origen, pero especialmente desde los huesos largos. Según las definiciones y la acuciosidad diagnóstica existe un amplio rango de incidencia (0,25-35\%)[83], aún cuando se pueden encontrar partículas de grasa en arteria pulmonar hasta en el $90 \%$ de los casos. La embolia grasa puede tener carácter fulminante 0 presentarse en forma subaguda y ocurrir 12 a $24 \mathrm{~h}$ después de la injuria. La fijación esquelética es una medida primordial para evitar la embolia grasa, pero si hay signos o sospecha clínica, debe plantearse la opción de fijación externa porque la manipulación de los canales medulares puede aumentar su incidencia[84],[85],[86].

En una lesión intratorácica o de otra localización debe buscarse la causa del shock, habitualmente hipovolémico. Si el shock se acompaña de presión venosa central elevada se debe descartar: neumotórax con o sin enfisema mediastínico, hemotórax, contusión miocárdica y taponamiento cardíaco.

Se deben plantear diversos conceptos asociados al paciente politraumatizado como la reanimación/resucitación de control de daños, el uso de ácido tranexámico dentro de las primeras tres horas de sangrado agudo severo[87],[88], la fórmula 1:1:1 de hemoderivados para la transfusión masiva o la administración de sangre total que es un concepto que está mostrando cada vez más aceptación[89],[90],[91]. En el ambiente bélico, el uso de plasma liofilizado se ha posicionado, principalmente por su mayor estabilidad temperatura dependiente en relación con el plasma fresco congelado[92],[93].

\section{Anestesia}

En términos generales, sólo 15\% de los pacientes con traumatismo torácico requiere cirugía. La toracotomía de salvataje es un tipo de enfrentamiento posible en casos de gran inestabilidad cardiorrespiratoria[94],[95], sin embargo, la efectividad de esta maniobra es más bien baja. En casos de herida penetrante con signos vitales presentes, la cirugía tiene una tasa de sobrevida de $21 \%$, la que baja a $11 \%$ si no hay signos de vida. Si se trata de un trauma cerrado las cifras son aún menores, alcanzando 4,6\% y $0,7 \%$ respectivamente, lo cual pone en entredicho su aplicación[96].

Por lo ya descrito, la condición del paciente en general es bastante diferente de aquellos pacientes de cirugía torácica electiva. Además, la técnica anestésica para este tipo de pacientes debe comprender todas las medidas consecuentes con el manejo del paciente hipovolémico o en shock. 


\section{Evaluación}

Debe limitarse a lo que la situación permita. Además, de los exámenes de rutina (hemograma, uremia, glicemia, protrombina) se debe contar con el estudio radiológico de tórax, que es indispensable para contar con un índice de las probables complicaciones, y de evaluar alguna patología pulmonar previa, aún cuando la presencia de complicaciones intratorácicas puede dificultar su interpretación. El análisis de los gases en sangre es el único estudio de función pulmonar posible.

El uso de la ecografía (point of care) es una herramienta muy útil y versátil y que debe considerarse por su facilidad de aplicación[35],[36], especialmente si la urgencia del caso exige un transporte rápido a pabellón.

Los otros medios de diagnóstico se emplean de acuerdo a la necesidad del caso clínico en cuestión. La tomografía presenta una gran sensibilidad en la pesquisa de lesiones, así como en la detección de hemotórax y neumotórax mínimo, que la radiografía es incapaz de diagnosticar. La broncoscopía tiene su lugar en la evaluación de la integridad de la vía aérea y en la limpieza de secreciones o elementos sólidos productos de aspiración de contenido gástrico o elementos presentes en la boca al momento del accidente. La ecocardiografía transesofágica representa un examen muy útil en la evaluación de estos pacientes ya que es un método no invasivo, rápido, capaz de entregar información sobre la condición hemodinámica (precarga y contractilidad), mostrar signos de contusión miocárdica y evaluar la integridad de la aorta.

\section{Monitorización}

Su instalación depende de la premura del caso. Si se recibe una emergencia, como una herida cardíaca por arma blanca, frecuentemente se está obligado al inicio rápido de la anestesia, no siendo posible la instalación de monitoreo invasivo, ya que la realización de procedimientos antes de la inducción sólo produce demora en la corrección quirúrgica y, en algunos casos, no son necesarios después de ésta. Así mismo hay casos en que el monitoreo básico estándar (ECG, presión arterial no invasiva, oxímetro de pulso, capnógrafo, termómetro) se debe instalar durante o incluso después de la inducción anestésica. Una sonda vesical se debe instalar en el momento que sea posible. Por todo esto, es necesario aprovechar al máximo lo que la clínica nos entrega: coloración de piel (palidez, cianosis), llene capilar, color de la sangre en el campo operatorio.
La instalación de una vía central siempre es posible una vez que la cirugía ha comenzado, utilizándose principalmente para la administración de drogas vasoactivas. No es una vía de alto flujo para la reposición de volumen y su utilidad como evaluación de la volemia es discutible per se y si el paciente es posicionado en decúbito lateral existe la duda agregada de establecer el punto cero.

\section{Técnica anestésica}

En situaciones de urgencia, el lugar de la anestesia regional está limitado a una técnica de analgesia postoperatoria o en lesiones parietales extensas, que por dolor afectan la mecánica ventilatoria y el reflejo de la tos. Resulta particularmente útil en los pacientes añosos con lesiones esqueléticas[4]. Raramente se puede realizar una técnica mixta con anestesia general, en el contexto de la cirugía torácica de urgencia.

Estos pacientes deben ser tratados como portadores de estómago lleno, utilizando todas las medidas descritas en tal caso para la inducción de la anestesia. Si existe un hemoneumotórax y ese hemitórax no va a ser abordado quirúrgicamente, se debe instalar un drenaje torácico antes o, aún mejor, durante la inducción anestésica.

Si las condiciones hemodinámicas son inestables, debe evitarse el uso de propofol. Como alternativas válidas de inducción se dispone de la ketamina, el etomidato y los opiáceos asociados a midazolam. La Tabla 8 muestra los efectos hemodinámicos de los distintos inductores[28]. En caso de neurotrauma concomitante está más indicado el uso de propofol o etomidato, sin embargo, la ketamina puede ser usada si el aumento de la presión intracraneana es compensado con la mantención o aumento de la presión arterial media y con ello de la presión de perfusión cerebral.

La mantención de la anestesia generalmente se realiza con gases halogenados, los que presentan varias ventajas: inhiben la irritabilidad de la vía aérea, pueden revertir la broncoconstricción, a diferencia del fentanilo tienen una eliminación rápida, lo que permite evaluar la condición ventilatoria del paciente en el posoperatorio inmediato y brindan una razonable estabilidad cardiovascular aún considerando pacientes cardiópatas o hipertensos. Una sugerencia de objetivo hemodinámico es lograr una presión arterial sistólica que no exceda el rango de 90 a $110 \mathrm{mmHg}$ [97].

La cirugía pulmonar y aquella sobre la aorta descendente se realizan a través de una toracotomía en posición decúbito lateral, en tanto que la cirugía sobre la aorta ascendente y el arco aórtico se abordan 
por esternotomía. La posición y abordaje en la cirugía por lesión de estructuras vasculares depende del vaso lesionado.

De acuerdo a la cirugía realizada sobre la aorta se debe instalar un bypass parcial pasivo, soporte cardiopulmonar (ECMO, del inglés ExtraCorporeal Membrane Oxygenation) o bien circulación extracorpórea franca con o sin paro circulatorio hipotérmico.

En decúbito lateral, como consecuencia de una suma de factores cuya revisión completa no es objeto de este artículo, se altera la distribución del flujo sanguíneo y la ventilación de cada pulmón. La consecuencia funcional es la producción de importantes alteraciones en la relación ventilación/perfusión (V/Q), con un pulmón superior que queda con una mayor ventilación y una menor perfusión, mientras que el pulmón inferior queda con una mayor perfusión y menor ventilación, habiendo una importante posibilidad de cierre de la vía aérea lo que, sumado al uso de una fracción inspirada de oxígeno alta, brinda las condiciones ideales para la formación de atelectasias por reabsorción. A esto se debe sumar el compromiso del parénquima pulmonar por el propio traumatismo y el efecto deletéreo de una eventual inestabilidad cardiovascular sobre la relación V/Q.

Ateniéndose a la lista de indicaciones absolutas o de alta prioridad, el uso de ventilación monopulmonar (VMP) en cirugía de urgencia pudiera restringirse a: disrupción traqueobronquial, fístula broncopleural o cutánea, sangrado pulmonar, lesiones de esófago, lesiones extensas de pulmón o de la pared, o lesiones vasculares. Sin embargo, la experiencia en el Hospital de Urgencia Asistencia Pública es que debe tenerse en cuenta el aislamiento pulmonar también en lesiones pulmonares menores, exploración por hemotórax, etc. Un campo quirúrgico quieto favorece la maniobra quirúrgica, sobre todo si se considera que el cirujano de urgencia no siempre está habituado a este tipo de procedimientos. En esta perspectiva, aunque se trate de una inducción en secuencia rápida, se debe pensar en primera instancia en la instalación de un tubo de doble lumen.

La ventilación mecánica intraoperatoria actualmente está muy influenciada por los conceptos de protección pulmonar provenientes de la medicina intensiva. La aplicación de este concepto protector durante la VMP ha ayudado a mejorar los resultados sobre la morbilidad. Se recomienda programar un volumen corriente menor de $7 \mathrm{ml} \cdot \mathrm{kg}^{-1}$, usar maniobras de reclutamiento y aplicar $5 \mathrm{cmH}_{2} \mathrm{O}$ o más de PEEP[98]. En un procedimiento bastante engorroso se ha propuesto buscar el PEEP que entregue la mejor distensibilidad (compliance) en cada paciente y así lograr la mejor presión de distensión (driving pressure) [99]. La frecuencia respiratoria se adecua para mantener la $\mathrm{PaCO}_{2}$ entre 35 y $40 \mathrm{mmHg}$. Para evitar en lo posible el barotrauma, se considera un límite de 35 $\mathrm{CmH}_{2} \mathrm{O}$ para la presión máxima (peak) y de $25 \mathrm{cmH}_{2} \mathrm{O}$ para la presión meseta (plateau).

En el contexto del politraumatizado hay condiciones en que la aplicación de PEEP debiera ponerse en duda, como en los casos de fístula broncopleural, neumotórax, presencia de broncoconstricción o contusión miocárdica[100].

Con la perspectiva de mantener la $\mathrm{PaO}_{2}$ cercana a $100 \mathrm{mmHg}$, la fracción inspirada de oxígeno se eleva en un inicio hasta $100 \%$ incluso a pesar del riesgo de atelectasias por reabsorción, sobre todo en casos de urgencia en que los pacientes ya presentan cierto compromiso respiratorio o hemodinámico. Con estos parámetros de ventilación lo habitual es que la oxigenación sea suficiente, pero la velocidad a la que cae la $\mathrm{PaO}_{2}$ en caso de apnea es el doble que con ventilación bipulmonar[101].

Es ideal que la cirugía pueda realizarse en decúbito lateral si se realizará VMP para aprovechar la contribución del efecto gravitatorio sobre la perfusión pulmonar, ya que en posición supina se observa una $\mathrm{PaO}_{2}$ mucho menor[102] junto a un shunt mayor (45\% versus $25 \%-28 \%$ )[103].

Tabla 8. Efectos hemodinámicos de los distintos inductores en situación de hipovolemia

\begin{tabular}{lcccccc}
\hline Agente & PAM & FC & RVS & $\begin{array}{c}\text { Venodilata- } \\
\text { ción }\end{array}$ & D. Cardiaco & Contractilidad \\
Etomidato & - & - & - & - & - & - \\
Ketamina & $\uparrow \uparrow$ & $\uparrow \uparrow$ & $\uparrow$ & - & $\uparrow$ & $\star \downarrow 0 \uparrow$ \\
Midazolam & $-\downarrow$ & $-\uparrow$ & $-\downarrow$ & $\uparrow$ & $-\downarrow$ & $\downarrow$ \\
Propofol & $\downarrow$ & $\uparrow$ & $\downarrow$ & $\uparrow$ & - & $\downarrow$ \\
\hline
\end{tabular}

PAM: presión arterial media; FC: frecuencia cardíaca; RVS: resistencia vascular sistémica; *: depende del nivel intrínseco de catecolaminas 
La vasoconstricción pulmonar hipóxica (VPH) es el fenómeno activo que ocurre en el pulmón no ventilado para derivar el flujo sanguíneo al pulmón ventilado y así disminuir el shunt, con un efecto máximo teórico del 50\%[104]. En el contexto del politraumatizado se presentan diversos factores descritos que afectan la eficacia de esta respuesta activa: el traumatismo mismo, la manipulación quirúrgica, hipertensión o hipotensión pulmonar, débito cardiaco bajo o hiperdinámi$\mathrm{co}$, uso de agentes anestésicos y drogas vasoactivas.

Los agentes anestésicos endovenosos no alteran la VPH en forma significativa. La ketamina, un inductor usado en el contexto del politraumatizado, es capaz de preservar la VPH en condiciones experimentales[105]. El propofol incluso potencia la respuesta vasoconstrictora a la hipoxia alveolar[34],[106], pero su efecto hemodinámico sistémico empeora esta respuesta. Los anestésicos inhalatorios halogenados inhiben la VPH en forma dosis dependiente[107],[108],[109],[110], con una aparente equipotencia tanto en experimentación[36] como en clínica[37],[38],[39] aún cuando algún estudio sea discrepante[111]. De todos modos, se puede afirmar que la inhibición de la VPH por 1 MAC de halogenado contribuye sólo con 4\%-5\% de aumento del shunt[112].

Las drogas vasoactivas tienen un efecto negativo sobre la VPH. La nitroglicerina, el nitroprusiato de sodio, los antagonistas del calcio, los agonistas beta 2 , la dobutamina, la dopamina, la epinefrina, la fenilefrina: todos ellos disminuyen la VPH derivando flujo sanguíneo hacia zonas no ventiladas, unos por vasodilatar zonas mal ventiladas, otros por vasocontraer zonas ventiladas, otros por alterar el débito cardíaco.

La hipoxemia durante la VMP es una situación estresante. Como primera causa se debe descartar la mala posición del tubo de doble lumen y conjuntamente se debe corregir posibles elementos causales como la $\mathrm{FiO}_{2}$, hipoventilación, inestabilidad hemodinámica (arritmias, hipotensión) o alguno de los factores que alteran la $\mathrm{VPH}$.

Si la corrección de todos los factores no permite mejorar la oxemia, hay dos técnica útiles: 1) el uso u

\section{Referencias}

1. Haberer JP, Chartier C, Féniès V, Faitg R, Dieng PN. Conduite á tenir initiale et orientation diagnostique et thérapeutique devant un traumatisé du thorax. In: Carli P, Ed. Les traumatismes graves. JEPU. Paris: Arnette, 1987. P. 73-93

2. Horton TG, Cohn SM, Heid MP et al. Identification of trauma patients at risk of thoracic aortic tear by me- optimización del PEEP en el pulmón ventilado, cuyo efecto es variable[113],[114] en función del estado pulmonar previo de cada paciente, el nivel de auto PEEP[115] y la CRF previa a la instalación de PEEP; sin embargo, debe evitarse niveles elevados de PEEP, ya que pueden empeorar la relación V/Q y provocar compromiso hemodinámico[116]; 2) el uso de 5 a 10 $\mathrm{CmH}_{2} \mathrm{O}$ de CPAP en el pulmón no ventilado que tiene un efecto de instalación lento, pero efectivo de aumento de la $\mathrm{PaO}_{2}$. Se ha descrito también el uso combinado de PEEP Y CPAP[117]. Se ha intentado también la ventilación a alta frecuencia[118] y el uso de óxido nítrico[119], sin resultados satisfactorios.

\section{Manejo del dolor}

El tratamiento del dolor posoperatorio es de trascendental importancia en esta cirugía, en especial con lesiones parietales producidas por el traumatismo. Una buena analgesia facilita la kinesiterapia respiratoria, evita el deterioro de la función pulmonar y permite una deambulación precoz, todo lo cual contribuye a disminuir las complicaciones pulmonares y tromboembólicas[4].

La terapia mutimodal es el eje de la analgesia dentro de la cual la técnica peridural se ha constituido en su eje central. El control del dolor alcanzado con esta técnica demuestra una amplia ventaja sobre la analgesia endovenosa tanto en efectividad, como en tiempo de estadía en intensivo y días en ventilación mecánica[120],[121],[122]. Existen otras técnicas regionales como el bloqueo del serrato, el bloqueo paravertebral o del erector espinal[123],[124],[125] que han ido demostrando su utilidad en cirugía electiva, en especial el bloqueo paravertebral que ha mostrado igual eficacia en la analgesia posoperatoria con algunas ventajas en la presentación de efectos colaterales menores. Sin embargo, en el contexto del trauma torácico no existen datos de efectividad comparada[126],[127]. De todas formas, al considerar la presencia de lesiones parietales bilaterales o del abdomen pareciera lógico privilegiar la técnica peridural.

chanism of injury. Journal of Trauma 2000;48:100813. https://doi.org/10.1097/00005373-20000600000003

3. Fegheli NT, Prisant LM. Blunt myocardial injury. Chest 1995;108:1673-7. https://doi.org/10.1378/ chest.108.6.1673

4. Watson RJ, Chir B: The anesthetic management of chest trauma. Problems in Anesthesia. 1990; Vol 4(3).

5. Chauhan R, Copeland CC, Murray M. Improvised 
explosive devices: anesthetic implications. Curr Anesthesiol Rep 2018; 8:71-77. https://doi. org/10.1007/s40140-018-0260-y

6. Ziegler DW, Agarwal NN. The morbidity and mortality of rib fractures. J Trauma 1994; 37:975-9. https://doi. org/10.1097/00005373199412000-00018

7. Bulger EM, Arneson MA, Mock ChN, Jurkovich GJ. Rib fractures in the elderly. Journal of Trauma 2000;48:1040-6. https:// doi.org/10.1097/00005373200006000-00007

8. Cohn SM. Pulmonary contusion: review of the clinical entity. J Trauma 1997;42:973-9. https:// doi.org/10.1097/00005373199705000-00033

9. Barnea $Y$, Kashtan H, Skornick $Y$, Werbin N. Isolated rib fractures in elderly patients: mortality and morbidity. Can J Surg 2002; 45:43-6.

10. Benjamin $E$, Recinos $G$, Aiolfi $A$ et al. Flail chest: less deadly than originally thought. World J Surg 2018; 42:3927-31. https://doi. org/10.1007/s00268-018-47236

11. Roberts S, Skinner D, Biccard $B$, Rodseth RN. The role of non-invasive ventilation in blunt chest trauma: systematic review and meta-analysis. Eur J Trauma Emerg Surg 2014;40:553-9. https://doi.org/10.1007/s00068013-0370-7

12. Parry NG, Moffat B, Vogt K. Blunt thoracic trauma: recent advances and outstanding questions. Curr Opin Crit Care. 2015;21:544-8. https://doi.org/10.1097/ MCC.0000000000000251

13. Cataneo AJM, Cataneo DC, de Oliveira FHS et al. Intervenciones quirúrgicas versus no quirúrgicas para el tórax inestable. Cochrane Database of Systematic Reviews. Disponible en: https://www.
cochrane.org/CD009919/INJ

14. Kasotakis $G$, Hasenboehler EA, Streib EW, et al. Operative fixation of rib fractures after blunt trauma: A practice management guideline from the Eastern Association for the Surgery of Trauma. J Trauma Acute Care Surg 2017; 82:618. https://doi.org/10.1097/ TA.0000000000001350

15. Bulger EM, Arneson MA, Mock CN, Jurkovich GJ. Rib fractures in the elderly. J Trauma 2000;48:1040. https:// doi.org/10.1097/00005373200006000-00007

16. Mackersie RC, Karagianes TG, Hoyt DB, Davis JW. Prospective evaluation of epidural and intravenous administration of fentanyl for pain control and restoration of ventilatory function following multiple rib fractures. J Trauma 1991; 31:443-9. https:// doi.org/10.1097/00005373199104000-00002

17. Ullman DA, Fortune JB, Greenhouse BB et al. The treatment of patients with multiple rib fractures using continuous thoracic epidural narcotic infusion. Reg Anesth 1989;14:43-7.

18. Bulger EM, Edwards T, Klotz $P$ et al. Epidural analgesia improves outcome after multiple rib fractures. Surgery 2004;136:42630. https://doi.org/10.1016/j. surg.2004.05.019

19. Galvagno SM, Smith $\mathrm{ChE}$, $M D$, Varon AJ et cols. Pain management for blunt thoracic trauma: A joint practice management guideline from the Eastern Association for the Surgery of Trauma and Trauma Anesthesiology Society. Trauma Acute Care Surg. 2016;81:93651. https://doi.org/10.1097/ TA.0000000000001209

20. Brookes JG, Dunn RJ, Rogers IR. Sternal fractures: a retrospective analysis of 272 cases. J
Trauma 1993;35:46-54. https:// doi.org/10.1097/00005373199307000-00008

21. Hills MW, Delprado AM, Deane SA. Sternal fractures: associated injuries and management. Journal of Trauma 1993;35:55-60. https://doi. org/10.1097/00005373199307000-00009

22. Harley DP, Mena I. Cardiac and vascular sequelae of sternal fractures. J Trauma 1986;26 553-5. https://doi. org/10.1097/00005373198606000-00011

23. Richardson JD, McElvein RB, Trinkle JK. First rib fracture: a hallmark of severe trauma. Ann Surg 1975;181:251-4. https:// doi.org/10.1097/00000658197503000-00001

24. Newman RJ, Jones IS. A prospective study of 413 consecutive car occupantswith chest injuries. Journal of Trauma 1984;24:129-35. https:// doi.org/10.1097/00005373198402000-00007

25. Crestanello JA, Samuels LE, Kaufman MS et al. Sternal Fracture with Mediastinal Hematoma: Delayed Cardiopulmonary Sequelae. Journal of Trauma 1999;47:161-4. https:// doi.org/10.1097/00005373199907000-00034

26. Brown CVR, Velmahor G, Wang $D$ et al. Association of scapular fractures and blunt thoracic injury: fact or fiction. Am Surg Assos 2005;71:54-7. https://doi./ 10.1177/000313480507100110

27. Karmy-Jones R, Jurkovich GJ, Shatz DV et al. Management of Traumatic Lung Injury: A Western Trauma Association Multicenter Review. J Trauma 2001;51:1049-53. https:// doi.org/10.1097/00005373200112000-00004

28. Kingsley ChP. Perioperative anesthetic management of 
thoracic trauma. Anesthesiol

Clin 1999;17:183-95. https://

doi.org/10.1016/S0889-

8537(05)70087-0

29. Devitt JH, McLean RF, Koch JP. Anaesthetic management of acute blunt thoracic trauma. Can J Anaesth 1991;38:50610. https://doi.org/10.1007/ BF03007590

30. Pape H-Ch, Remmers D, Rice J et al. Appraisal of early evaluation of blunt chest trauma: development of a standardized scoring system for initial clinical decision making. J Trauma 2000;49:496-504. https:// doi.org/10.1097/00005373200009000-00018

31. Miller PR, Croce MA, Bee TK, et al. ARDS after pulmonary contusion: accurate measurement of contusion volume identifies high-risk patients. J Trauma 2001;51:223-8. https:// doi.org/10.1097/00005373200108000-00003

32. Soldati G, Testa A, Silva FR, et al. Chest ultrasonography in lung contusion. Chest 2006; 130:5338. https://doi.org/10.1378/ chest.130.2.533

33. Ball CG, Ranson MK, RodriguezGalvez M, et al. Sonographic depiction of posttraumatic alveolar-interstitial disease: the hand-held diagnosis of a pulmonary contusion. J Trauma 2009; 66:962. https://doi.org/10.1097/ TA.0b013e3180600949

34. McCunn M, Mauritz W, Sutcliffe A. Guidelines for management of mechanical ventilation in critically injured patients. 2008. ITACCS Management of Mechanical Ventilation in Critically Injured Patients. In C. Smith (Ed.), Trauma Anesthesia (pp. 465-470). Cambridge: Cambridge University Press.

35. Zahran T El, Sayed MJ El. Prehospital ultrasound in trauma: a review of current and potential future clinical applications. J Emerg Trauma Shock 2018; 11: 4-9. https://doi.org/10.4103/ JETS.JETS_117_17

36. Gleeson T, Blehar D. Pointof-care ultrasound in trauma. Semin Ultrasound CT MR 2018; 39: 374-83. https://doi. org/10.1053/j.sult.2018.03.007

37. Mowery NT, Gunter OL, Collier $\mathrm{BR}$, et al. Practice management guidelines for management of hemothorax and occult pneumothorax. J Trauma 2011; 70:510. https://doi.org/10.1097/ TA.0b013e31820b5c31

38. Huang WY, Lu IY, Yang Ch, et cols. Efficiency analysis of direct video-assisted thoracoscopic surgery in elderly patients with blunt traumatic hemothorax without an initial thoracoscopic. BioMed research international. Volumen 2016, Article ID 3741426, 7 pages http://dx.dir. org/10.1155/2016/3741426

39. Paci M, Ferrari G, Annessi $V$, et cols. The role of diagnostic VATS in penetrating thoracic injuries. World Journal of Emergency Surgery 2006;1:30 https://doi. org/10.1186/1749-7922-1-30

40. Bertinchant JP, Polge A, Mohty D et al. Evaluation of incidence, clinical significance, and prognostic value of circulating cardiac troponin I and T elevation in hemodynamically stable patients with suspected myocardial contusion after blunt chest trauma. J Trauma 2000; 48: 924-31. https:// doi.org/10.1097/00005373200005000-00018

41. Synbrandy KC, Cramer MJM, Burgersdijk C. Diagnosing cardiac contusion: old wisdom and new insights. Heart 2003; 89: 485-9. https://doi.org/10.1136/ heart.89.5.485

42. Maron BJ, Estes NA 3rd. Commotio cordis. N Engl J Med 2010; 362:917 https://doi. org/10.1056/NEJMra0910111
43. Rodríguez-González F, Martínez-Quintana E. Cardiogenic shock following blunt chest trauma. J Emerg Trauma Shock 2010; 3:398. https://doi. org/10.4103/0974-2700.70772

44. Bansal MK, Maraj S, Chewaproug $D$ et al. Myocardial contusion injury: redefining the diagnostic algorithm. Emerg Med J 2005; 22: 465-9. https://doi. org/10.1136/emj.2004.015339

45. Chirillo F, Totis O, Cavarzerani A et al. Usefulness of transthoracic and transoesophageal echocardiography in recognition and management of cardiovascular injuries after blunt chest trauma. Heart 1996; 75: 301-6. https:// doi.org/10.1136/hrt.75.3.301

46. Ferjani M, Droc G, Dreux S et al. Circulating Cardiac Troponin T in Myocardial Contusion. Chest 1997; 111: 427-33. https://doi. org/10.1378/chest.111.2.427

47. Orliaguet $G$, Ferjani $M$, Riou B. The Heart in Blunt Trauma. Anesthesiology 2001; 95: 544-8. https:// doi.org/10.1097/00000542200108000-00041

48. Holanda MS, Domínguez MJ, López-Espadas F, et al. Cardiac contusion following blunt chest trauma. Eur J Emerg Med 2006; 13:373. https://doi.org/10.1097/ MEJ.0b013e32801112f6

49. Wall MJ, Mattox KL, Chen ChD, Baldwin JC. Acute Management of Complex Cardiac Injuries. J Trauma 1997; 42: 905-12. https:// doi.org/10.1097/00005373199705000-00022

50. Degiannis E, Loogna P, Doll D et al. Penetrating Cardiac Injuries: Recent Experience in South Africa. World J Surg 2006; 30:125864. https://doi.org/10.1007/ s00268-005-0463-5

51. Parmley LF, Mattingly TW, Manion WC et al. Non penetrating traumatic injury of the aorta. 
Circulation 1958; 17: 1086-100. https://doi.org/10.1161/01. CIR.17.6.1086

52. Richens D, Kotidis K, Neale M et al. Rupture of the aorta following road traffic accidents in the United Kingdom 1992-1999. The results of the co-operative crash injury study. Eur J Cardiothorac Surg 2003; 23: 143-8. https://doi.org/10.1016/S10107940(02)00720-0

53. Feczko JD, Lynch L, Pless JE et al. An autopsy case review of 142 nonpenetrating (blunt) injuries of the aorta. J Trauma 1992; 33: 846-9. https:// doi.org/10.1097/00005373199212000-00009

54. Fabian TC, Richardson JD, Croce MA et al. Prospective study of blunt aortic injury: multicenter trial of the american association for the surgery of trauma. J Trauma 1997; 42: 374-83. https:// doi.org/10.1097/00005373199703000-00003

55. Williams JS, Graff JA, Uku JM, Steinig JP. Aortic Injury in Vehicular Trauma. Thorac Surg 1994;57:726-30. https:// doi.org/10.1016/00034975(94)90576-2

56. Lee J, Harris JH, Duke JH, Scott Williams J. Noncorrelation between thoracic skeletal injuries and acute traumatic aortic tear. J Trauma 1997; 43: 400-4. https:// doi.org/10.1097/00005373199709000-00002

57. Woodring JH, Dillon ML. Radiographic manifestations of mediastinal hemorrhage from blunt chest trauma. Ann Thorac Surg 1984; 37: 171-9. https://doi.org/10.1016/S00034975(10)60311-6

58. Stark P. Progress in clinical radiology. Invest Radiol 1990; 25: 1265-75. https:// doi.org/10.1097/00004424199011000-00024

59. Lichtenberger JP, Kim AM, Fisher
D et al. Imaging of combatrelated thoracic trauma - blunt trauma and blast lung injury. Mil Med 2018; 183: e89-e96. https://doi.org/10.1093/milmed/ usx033

60. Patterson BO, Holt PJ, Cleanthis $\mathrm{M}$ et al. Imaging vascular trauma. Br J Surg 2012; 99: 494505. https://doi.org/10.1002/ bjs. 7763

61. Kaewlai R, Avery LL, Asrani AV, Novelline RA. Multidetector CT of blunt thoracic trauma. Radiographics 2008; 28: 155570. https://doi.org/10.1148/ rg. 286085510

62. Miller FB, Richardson JD, Thomas $\mathrm{HA}$ et al. Role of CT in diagnosis of major arterial injury after blunt thoracic trauma. Surgery 1989; 106: 596-603.

63. McLean TR, Olinger GN, Thorsen MK. Computed tomography in the evaluation of the aorta in patients sustaining blunt chest trauma. J Trauma 1991; 31: 254-6. https:// doi.org/10.1097/00005373199131020-00018

64. Tomiak MM, Rosenblum JD, Messersmith RN, Zarins CK. Use of CT for diagnosis of traumatic rupture of the thoracic aorta. An Vascular Surg 1993; 7: 130-9. https://doi.org/10.1007/ BF02001006

65. Dyer DS, Moore EE, Ilke DN et cols. Thoracic aortic injury: how predictive is mechanism and is chest computed tomography a reliable screening tool? A prospective study of 1561 patients. J Trauma 2000; 48: 673-83. https:// doi.org/10.1097/00005373200004000-00015

66. Fabian TC, Davis KA, Gavant $\mathrm{ML}$ et al. Prospective study of blunt artic injury. Ann Surg 1998; 22: 666-77. https:// doi.org/10.1097/00000658199805000-00007
67. Subramaniam K, Subramaniam B. Role of Echocardiography in Aortic Surgery. En: Anesthesia and Perioperative Care for Aortic Surgery, páginas 47-97. 2011 Springer Science+Business Media New York. https://doi. org/10.1007/978-0-387-859224_4

68. Wahl WL, Michaels AJ, Wang SC, et cols. Blunt thoracic aortic injury: delayed or early repair? Journal of Trauma 1999;47:254-60. https:// doi.org/10.1097/00005373199908000-00006

69. von Oppell UO, Dunne TT, De Groot MK, Zilla P. Traumatic aortic rupture: twenty year metaanalysis of mortality and risk of paraplegia. Ann Thor Surg 1994; 58: 585-93. https://doi.org/10.1016/00034975(94)92270-5

70. McCunn M, Gordon E, Scott Th. Anesthetic Concerns in Trauma Victims Requiring Operative Intervention: The Patient Too Sick to Anesthetize. Anesthesiol Clin 2010; 28: 97116. https://doi.org/10.1016/j. anclin.2010.01.004

71. Xenos ES, Abedi NN, Davenport DL et al. Meta-analysis of endovascular vs open repair for traumatic descending thoracic aortic rupture. J Vasc Surg 2008; 48: 1343-51. https://doi. org/10.1016/j.jvs.2008.04.060

72. Tang GL, Tehrani HY, Usman A et al. Reduced mortality, paraplegia, and stroke with stent graft repair of blunt aortic transactions: a modern metaanalysis. J Vasc Surg 2008; 47: 671-5. https://doi.org/10.1016/j. jvs.2007.08.031

73. Urgnani F, Lerut $P$, Da Rocha $M$ et al. Endovascular treatment of acute traumatic thoracic aortic injuries: a retrospective analysis of 20 cases. J Thorac Cardiovasc Surg 2009; 138: 1129- 
38. https://doi.org/10.1016/j. jtcvs.2008.10.057

74. Walsh SR, Tang TY, Sadat U et al. Endovascular stenting versus open surgery for thoracic aortic disease: systemic review and meta-analysis of perioperative results. J Vasc Surg 2008; 47: 1094-8. https://doi. org/10.1016/j.jvs.2007.09.062

75. Parry NG, Moffat $B$, Vogt K. Blunt thoracic trauma: recent advances and outstanding questions. Curr Opin Crit Care. 2015; 21: 544-8. https://doi.org/10.1097/ MCC.0000000000000251

76. Pang D, Hildebrand D, Bachoo $P$. Thoracic endovascular repair (TEVAR) versus open surgery for blunt traumatic thoracic aortic injury (Review). Cochrane Database Syst Rev. 2105 Sep 25;(9).

77. Cassada DC, Munyikwa MP, Moniz MP et al. Acute injuries of the trachea and major bronchi: importance of early diagnosis. Ann Thor Surg 2000; 69: 1563-7. https://doi.org/10.1016/ S0003-4975(00)01077-8

78. Pilcher JA, Ishitani MB, Rodgers BM. Left upper lobe bronchus reimplantation for nonpenetrating horacic trauma. Ann Thor Surg 2000; 69: 273-5. https://doi.org/10.1016/S00034975(99)01207-2

79. Vassallo J, Smith JE, Bouam. WaIlis LA. The civilian validation of the Modified Physiological Triage Tool (MPTT) - an evidence-based approach to primary major incident triage James. J R Army Med Corps 2017; 163: 383-7. https://doi.org/10.1136/jramc-2017-000771

80. Vassallo J, Horne S, Smith JE. Triage and the Modified Physiological Triage Tool-24 (MPTT-24). J R Army Med Corps 2018; 24: 1-4. https://doi.org/10.1136/ jramc-2017-000878

81. Hudson AJ, Strandenes G,
Bjerkvig CK et al. Airway and ventilation management strategies for hemorrhagic shock. To tube, or not to tube, that is the question! J Trauma Acute Care Surg 2018; 84: S77S82. https://doi.org/10.1097/ TA.0000000000001822

82. Hoffmann M, Czorlich P, Lehmann $W$ et al. The impact of prehospital intubation with and without sedation on outcome in trauma patients with a GCS of 8 or less. J Neurosurg Anesthesiol 2017; 29: 1617. https://doi.org/10.1097/ ANA.0000000000000275

83. Peltier LF. Fat embolism. A current concept. Clin Orthop 1969; 66: 241-53. https:// doi.org/10.1097/00003086196909000-00028

84. Akhtar S. Fat embolism. Anesthesiol Clin 2009; 27: 53350. https://doi.org/10.1016/j. anclin.2009.07.018

85. Heine TA, Halambeck BL, Mark JB. Fatal pulmonary fat embolism in the early postoperative period. Anesthesiology 1998; 89: 1589-91. https:// doi.org/10.1097/00000542199812000-00046

86. Djelouah I, Lefevre G, Ozier $Y$ et al. Fat embolism in orthopedic surgery: role of bone marrow fatty acid. Anesth Analg 1997; 85: 441-3. https:// doi.org/10.1213/00000539199708000-00035

87. Shakur H, Roberts I, Bautista R et al. Effects of tranexamic acid on death, vascular occlusive events and blood transfusion in trauma patients with significant hemorrhage (CRASH-2): a randomised placebo-controlled trial. Lancet 2010; 376: 23-32. https://doi.org/10.1016/S01406736(10)60835-5

88. Gayet-Ageron A, PrietoMerino D, Ker K et al. Effect of treatment delay on the effectiveness and safety of antifibrinolytics in acute severe haemorrhage: a meta-analysis of individual patient-level data from 40138 bleeding patients. Lancet 2017; 391: 125-32. https://doi.org/10.1016/S01406736(17)32455-8

89. Spinella PC, Perkins JG, Grathwohl KW et al. Warm fresh whole blood is independently associated with improved survival for patients with combatrelated traumatic injuries. J Trauma 2011; 66: 69-76. https://doi.org/10.1097/ TA.0b013e31819d85fb

90. Murdock AD, Berseus O, Hervig $\mathrm{T}$, et cols. Whole blood: the future of traumatic hemorrhagic shock resuscitation. Shock 2014; 41 (Supl 1): 62-69. https://doi.org/10.1097/ SHK.0000000000000134

91. Cap AP, Beckett A, Benov A et al. Whole blood transfusion. Mil Med 2018; 183 (Supl 2): 44-51. https://doi.org/10.1093/milmed/ usy 120

92. Stancil SA. Development of a new infusion protocol for austere trauma resuscitations. Air Med J 2017; 36: 239-43. https://doi. org/10.1016/j.amj.2017.02.006

93. Garrigue D, Godier A, Glacet A et al. French lyophilized plasma versus fresh frozen plasma for the initial management of trauma-induced coagulopathy: a randomized open-label trial. J Thromb Haemost 2018; 16: 481-9. https://doi.org/10.1111/ jth. 13929

94. Seamon MJ, Haut ER, Van Arendonk K et al. An evidencebased approach to patient selection for emergency department thoracotomy: a practice managementguideline from the Eastern Association for the Surgery of Trauma. J Trauma Acute Care Surg 2015; 79: 159-73. https://doi.org/10.1097/ 
TA.0000000000000648

95. Suzuki K, Inoue S, Morita S et al. Comparative effectiveness of emergency resuscitative thoracotomy versus closed chest compressions among patients with critical blunt trauma: a nationwide cohort study in Japan. PLoS One 2016; 11: 1-12. https://doi.org/10.1371/journal. pone. 0145963

96. DuBose J, Fabian T, Bee T et al. Contemporary utilization of resuscitative thoracotomy: results from the AAST aortic occlusion for resuscitation in trauma and acute care surgery (AORTA) multicenter registry. Shock 2018; 50: 414-20. https://doi.org/10.1097/ SHK.0000000000001091

97. Woolley T, Thompson P, Kirkman E et al. Trauma Hemostasis and Oxygenation Research Network position paper on the role of hypotensive resuscitation as part of remote damage control resuscitation. J Trauma Acute Care Surg 2018; 84 (Sup): S3S13. https://doi.org/10.1097/ TA.0000000000001856

98. Batchelor T, Rasbum N, Abdelnour-Berchtold E, Brunelli A et cols. Guidelines for enhanced recovery after lung surgery: recommendations of the Enhanced Recovery After Surgery (ERAS) Society and the European Society ofhoracic Durgery (ESTS). European Journal of Cardio-Thoracic Surgery 2019; 55: 91-115. https://doi.org/10.1093/ejcts/ ezy301

99. Park M, Ahn H, Kim JA et cols. Driving pressure during thoracic surgery. Anesthesiology 2019;130:385-93. https://doi.org/10.1097/ ALN.0000000000002600

100. Schulman DS, Biondi JW, Matthay RA, et al. Effect of positive end-expiratory pressure on right ventricular performance. Importance of baseline right ventricular function. Am J Med 1988; 84:5.7. https:// doi.org/10.1016/00029343(88)90009-5

101. Baraka A, Aouad M, Taha S et als. Apnea-induced hemoglobin desaturation durinh one-lung vs two-lung ventilation. Can J Anaesth 2000; 47: 58-61. https://doi.org/10.1007/ BF03020734

102. Bardoczky GI, Szegedi LL, d'Hollander AA et al. Two-lung and one-lung ventilation in patients with chronic obstructive pulmonary disease: the effects of position and $\mathrm{FiO} 2$ Anesth Analg 2000; 90: 35-41. https:// doi.org/10.1097/00000539200001000-00008

103. Watanabe S, Noguchi E, Yamada $S$ et al. Sequential changes of arterial oxygen tensión in the supine position during one-lung ventilation. Anesth Analg 2000; 90: 28-34. https:// doi.org/10.1097/00000539200001000-00007

104. Marshall BE, Marshall C. Continuity of response to hypoxic pulmonary vasoconstriction. Journal Apply Physiology 1980; 49: 189-96. https://doi.org/10.1152/ jappl.1980.49.2.189

105. Nakayama S, Murray PA. Ketamine preserves and propofol potentiates hypoxic pulmonary vasoconstriction compared with the conscious state in chronically instrumented dogs. Anesthesiology 1999; 91: 760-71. https:// doi.org/10.1097/00000542199909000-00029

106. Karzai W, Haberstroh J, Priebe $\mathrm{H}-\mathrm{J}$. Effects of desflurane and proppofol on arterial oxygenation during one-lung ventilation in the pig. Acta Anaesthesiol Scand 1998; 42: 648-52. https://doi. org/10.1111/j.1399-6576.1998. tb05296.x

107. Marshall C, Lindgren $L$, Marshall
BE. Effects of halothane, enflurane and isoflurane on hypoxic pulmonary vasoconstriction in rats lungs in vitro. Anesthesiology 1984; 60: 304-8. https:// doi.org/10.1097/00000542198404000-00006

108. Pagel PS, Fu JL, Damsk MC et al. Desflurane and isoflurane produce similar alterations in systemic and pulmonary hemodynamics and arterial oxygenation in patients undergoing one-lung ventilation durng thoracotomy. Anesth Analg 1998; 87: 800-7. https:// doi.org/10.1213/00000539199810000-00011

109. Wang JYY, Russell GN, Page $\mathrm{RD}$ et al. A comparison of the effects of desflurane and isoflurane on arterial oxygenation during one-lung ventilation. Anaesthesia 2000; 55: 163-83. https://doi.org/10.1046/j.13652044.2000.055002167.x

110. Wang JYY, Russell NG, Page RD et al. Comparison of the effects of sevoflurane and isoflurane on arterial oxygenation during one lung ventilation. $\mathrm{Br} J$ Anaeshe 1998; 81: 850-3. https://doi. org/10.1093/bja/81.6.850

111. Slinger $P$, Scott WAC. Arterial oxygenation of enflurane and isoflurane. Anesthesiology 1995; 82: 940-6. https://doi. org/10.1097/00000542199504000-00017

112. Benumof JL, Augustine SD, Gibbons JA. Halothane and isoflurane only slightly impair arterial oxygenation during one-lung ventilation in patients undergoing thoracotomy. Anesthesiology 1987; 67: 910-15. https:// doi.org/10.1097/00000542198712000-00006

113. Capan LM, Turndorf H, Patel C et al. Optimization of arterial oxygenation during one-lung anesthesia. Anesth Analg 1980; 59: 847-51. https:// 
doi.org/10.1213/00000539198011000-00007

114. Aalto-Setälä M, Heinonen J, Salorinne Y. Cardiorespiratory function durign thoracic anaesthesia: a comparison of two-lung ventilation ond one-lung ventilation with and without PEEP5. Acta Anaesthesiol Scand 1975; 19: 287-95. https://doi. org/10.1111/j.1399-6576.1975. tb05185.x

115. Inomata S, Nishikawa T, Saito S, Kihara S. "Best" PEEP during one-lung ventilation. British Journal of Anaesthesia 1997; 78: 745-56. https://doi.org/10.1093/ bja/78.6.754

116. Connery LE, Deignan MJ, Gujer MW, Richardson MG. Cardiovascular collapse associated with extreme iatrogenic PEEPi in patients with obstructive airways disease. Br J Anaesth 1999; 83: 493-5. https://doi.org/10.1093/ $\mathrm{bja} / 83.3 .493$

117. Cohen E, Eisenkraft JB, Thys DM et al. Oxygenation and hemodynamic changes during one-lug ventilation: effects of CPAP10, PEEP10 and CPAP10/PEEP10. J Cardiothorac Anesth 1988; 2: 34-40. https://doi.org/10.1016/08886296(88)90145-7
118. den Hoed PT, LeendertseVerloop K, Bruining HA, Bonjer HJ. Comparison of one-lung ventilation and high frequency ventilation in thoracoscopic surgery. Eur J Surg 1999; 165: 1031-4. https://doi./10.1080/110 241599750007847

119. Fradj K, Samain E, Delefosse D et al. Placebo-controlled study of inhaled nitric oxide to treat hypoxaemia during one-lung ventilation. Br J Anaesth 1999; 82: 208-12. https://doi.org/10.1093/ $\mathrm{bja} / 82.2 .208$

120. Zein Eldina A, Mohamedb M, Abdelmotelbb M, Abdelsabourb M. Comparison of thoracic epidural analgesia versus parenteral analgesia for traumatic multiple rib fractures. Menoufia Med J 2018; 31: 199-204.

121. Samet R, Slade I. Regional anesthesia for the acute trauma patient. Curr Anesthesiol Rep 2018; 8: 94-106. https://doi. org/10.1007/s40140-018-0254-9

122. B€uttner B, Mansur A, Kalmbach $M$, et al. Prehospital ultrasound-guided nerve blocks improve reduction-feasibility of dislocated extremity injuries compared to systemic analgesia. A randomized controlled trial. PLoS One 2018; 13: 1-12. https://doi.org/10.1371/journal. pone.0199776

123. Durant E, Dixon B, Luftig J et al. Ultrasound-guided serratus plane block for ED rib fracture pain control. Am J Emerg Med 2017; 35: 197.e3-197.e6. https://doi. org/10.1016/j.ajem.2016.07.021

124. Luftig J, Mantuani D, Herring AA et al. Successful emergency pain control for posterior rib fractures with ultrasound-guided erector spinae plane block. Am J Emerg Med 2018; 36: 13916. https://doi.org/10.1016/j. ajem.2017.12.060

125. Malekpour M, Hashmi A, Dove J,et al. Analgesic choice in management of rib fractures: paravertebral block or epidural analgesia? Anesth Analg 2017; 124: 1906-111. https://doi.org/10.1213/ ANE.0000000000002113

126. Thiruvenkatarajan $\mathrm{V}$, Cruz Eng $\mathrm{H}$, Adhikary SD. An update on regional analgesia for rib fractures. Curr Opin Anaesthesiol 2018; 31:601. https://doi.org/10.1097/ AC0.0000000000000637

127. El-Boghdadly K, Wiles MD. Regional anaesthesia for rib fractures: too many choices, too little evidence. Anaesthesia 2019; 74:564. https://doi.org/10.1111/ anae. 14634 